\title{
Sunspot number recalibration: The $\sim 1840-1920$ anomaly in the observer normalization factors of the group sunspot number
}

\author{
Edward W. Cliver* \\ National Solar Observatory, Boulder, CO 80303 USA \\ ${ }^{*}$ Corresponding author: ecliver@nso.edu
}

Received 3 May 2016 / Accepted 28 March 2017

\begin{abstract}
We analyze the normalization factors ( $k^{\prime}$-factors) used to scale secondary observers to the Royal Greenwich Observatory (RGO) reference series of the Hoyt \& Schatten (1998a, 1998b) group sunspot number (GSN). A time series of these $k^{\prime}$-factors exhibits an anomaly from 1841 to 1920 , viz., the average $k^{\prime}$-factor for all observers who began reporting groups from 1841 to 1883 is 1.075 vs. 1.431 for those who began from 1884 to 1920, with a progressive rise, on average, during the latter period. The 1883-1884 break between the two subintervals occurs precisely at the point where Hoyt and Schatten began to use a complex daisy-chaining method to scale observers to RGO. The 1841-1920 anomaly implies, implausibly, that the average sunspot observer who began from 1841 to 1883 was nearly as proficient at counting groups as mid-20th century RGO (for which $k^{\prime}=1.0$ by definition) while observers beginning during the 1884-1920 period regressed in group counting capability relative to those from the earlier interval. Instead, as shown elsewhere and substantiated here, RGO group counts increased relative to those of other long-term observers from 1874 to $\sim 1915$. This apparent inhomogeneity in the RGO group count series is primarily responsible for the increase in $k^{\prime}$-factors from 1884 to 1920 and the suppression, by $44 \%$ on average, of the Hoyt and Schatten GSN relative to the original Wolf sunspot number (WSN) before $\sim 1885$. Correcting for the early "learning curve" in the RGO reference series and minimizing the use of daisy-chaining rectifies the anomalous behavior of the $k^{\prime}$-factor series. The resultant GSN time series (designated GSN $\left.{ }^{*}\right)$ is in reasonable agreement with the revised WSN $\left(S_{N}{ }^{*}\right.$; Clette \& Lefèvre 2016) and the backbone-based group sunspot number $\left(R_{\mathrm{GS}}\right.$; Svalgaard \& Schatten 2016) but significantly higher than other recent reconstructions (Friedli, personal communication, 2016; Lockwood et al. 2014a, 2014b; Usoskin et al. 2016a). This result is substantiated by a "correction-factor" (CF) time series defined as the ratio of annual group counts of the Hoyt \& Schatten (1998a, 1998b) series to the average raw (unscaled) group counts of all observers, as well as by a comparison of the GSN and GSN* time series with a recent reconstruction of solar wind $B$ from 1845 to the present. The $\sim 1840-1920 k^{\prime}$-factor anomaly and its impact on the Hoyt and Schatten GSN are discussed in the context of the ongoing effort to recalibrate the sunspot number time series.
\end{abstract}

\section{Introduction}

Recently, much attention has been focused on the sunspot number time series (e.g., Clette et al. 2014; Lockwood et al. 2014a, 2014b; Usoskin et al. 2016a, 2016b; Cliver \& Ling 2016; Svalgaard \& Schatten 2016; Clette \& Lefëvre 2016; Cliver 2016; Lockwood et al. 2016a, 2016b). The original sunspot number was developed by Rudolf Wolf in the second half of the 19th century (Wolf 1851, 1856). This time series went unchallenged as the principal measure of solar activity until the 1990s when Hoyt and colleagues (Hoyt et al. 1994; Hoyt \& Schatten 1998a, 1998b) introduced the group sunspot number (GSN). Hoyt and Schatten significantly expanded the sunspot number database and thus were able to extend the GSN back to the earliest observations of sunspots in 1610 vs. the 1700 start year for the Wolf sunspot number (WSN) time series.

From 1700 to 1885 the WSN is, on average, $44 \%$ higher than the GSN (Fig. 1a; Cliver \& Ling 2016). This significant difference between the two time series, and the lack of consensus as to which is more correct, provided the principal motivation for a systematic examination from 2011 to 2015 of both the WSN and GSN time series (Cliver et al. 2013, 2015; Clette et al. 2014), termed the Sunspot Number Workshops. This effort resulted in the adoption of a revised WSN series (designated $S_{N}$; Clette \& Lefèvre 2016) in July 2015 by the World
Data Center for the Sunspot Index and Long-term Solar Observations at the Royal Observatory of Belgium (Clette et al. 2015 ) and the construction of a new group sunspot number series $\left(R_{\mathrm{GS}} ;\right.$ Svalgaard \& Schatten 2016). Both $S_{N}$ and $R_{\mathrm{GS}}$ hew closer to the original WSN than they do to the Hoyt \& Schatten (1998a, 1998b) GSN. The Sunspot Number Workshops and the publication of these two series fostered much interest and activity regarding the sunspot number, culminating in the proposal of three independently derived alternative time series: $R_{\mathrm{WL}}$ (Lockwood et al. 2014a, 2014b, 2016b), $R_{\mathrm{GU}}$ (Usoskin et al. 2016a), and $R_{\mathrm{WF}}$ (Friedli, personal communication, 2016), ${ }^{1}$ that more closely resemble GSN than $S_{N}$. Thus, in the present situation, we have a variant of the discrepancy that motivated the 2011-2015 examination of the discordant WSN and GSN time series. Instead of a disparity between two individual series (WSN and GSN), we now have a divergence between two groups of sunspot number series - WSN, $S_{N}$, and $R_{\mathrm{GS}}$ on the one hand and GSN, $R_{\mathrm{WL}}, R_{\mathrm{GU}}$, and $R_{\mathrm{WF}}$ on the other, both of which employ a variety of methodologies. In broad brush, the $R_{\mathrm{WL}}, R_{\mathrm{GU}}$, and $R_{\mathrm{WF}}$ series are slightly higher after $\sim 1950$ and significantly lower before $\sim 1880$ than $S_{N}$ and $R_{\mathrm{GS}}$ (Fig. 1b). The new group series in Figure $1 \mathrm{~b}$ are

\footnotetext{
${ }^{1}$ Here the initial subscript indicates whether the original Wolf $(\mathrm{W})$ prescription for the sunspot number, based on both group and spot counts, or the alternative Hoyt \& Schatten (1998a, 1998b) formulation, based only on groups $(\mathrm{G})$, was used.
} 

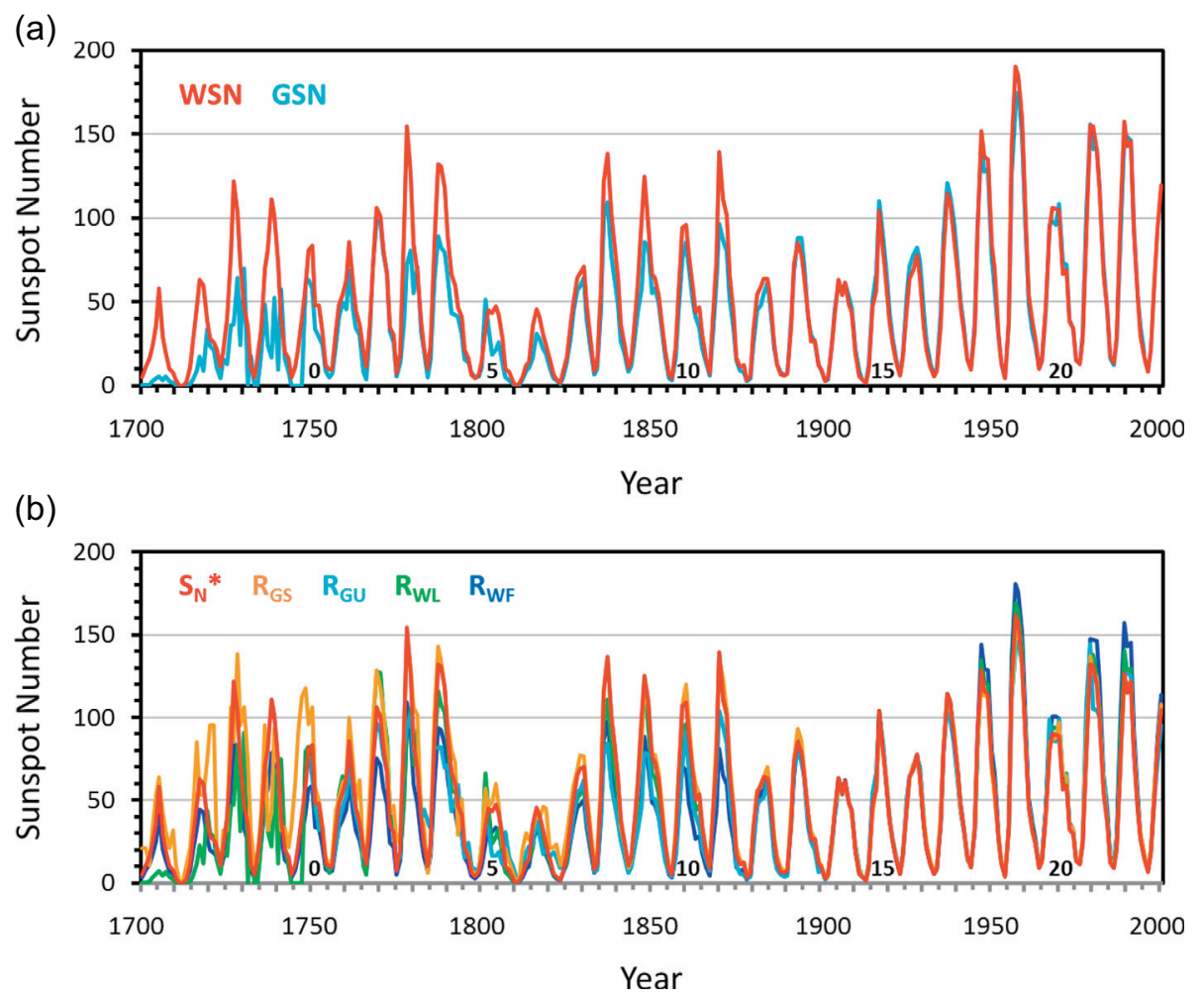

Fig. 1. (a) The Wolf (WSN) and Hoyt \& Schatten (1998a, 1998b) Group (GSN) sunspot number time series, for their 1700-1995 period of overlap. Cycle numbers are given at the bottom of the plot. (b) The two groupings of new sunspot number time series: (1) $S_{N}{ }^{*}$ (Clette \& Lefèvre 2016) and $R_{\mathrm{GS}}$ (Svalgaard \& Schatten 2016); and (2) $R_{\mathrm{WL}}$ (Lockwood et al. 2014a, 2014b, 2016b), $R_{\mathrm{GU}}$ (Usoskin et al. 2016a), and $R_{\mathrm{WF}}$ (Friedli, personal communication, 2016). Cycle numbers are given at the bottom of the plot.

normalized to $S_{N}{ }^{*}$ over the 1916-1946 interval where $S_{N} *=0.6 \times S_{N}$ to facilitate comparison with Figure 1a (see Clette \& Lefèvre 2016) and the 1916-1946 interval is chosen to avoid inhomogeneities in the original WSN and GSN time series (Clette et al. 2014; Cliver \& Ling 2016).

Because of the importance of the sunspot number in solar and solar-terrestrial physics, it is necessary that the differences between the various new time series be understood and reconciled. Thus at the recent Space Climate Symposium in Levi, Finland (http://www.spaceclimate.fi/SC6/), it was decided to continue with the work of the Sunspot Number Workshops. In this paper, we focus on a rather glaring peculiarity of the Hoyt \& Schatten (1998a) normalization factors ( $k^{\prime}$-factors) used to bring all observers to a common scale that - had it been noticed when the group sunspot number series was introduced - would have challenged its validity at the outset. A more limited examination of the Hoyt \& Schatten (1998a, 1998b) $k^{\prime}$-factors was presented in Cliver \& Ling (2016).

In addition to the criticism of the Hoyt \& Schatten (1998a, 1998b) observer normalization procedure presented here, Lockwood, Usoskin, and colleagues (Lockwood et al. 2016a, 2016b; Usoskin et al. 2016a, 2016b) have pointed out several potential problems in the methodology of the Svalgaard \& Schatten (2016) time series, some of which (linear regression, direct proportionality, comparison of observers over entire intervals of overlap, daisy-chaining) apply more generally to all of the time series in Figures 1 and 2, including the Hoyt and Schatten series, but excepting the Usoskin et al. (2016) series which employs a novel normalization procedure based on a synthetic primary observer and non-linear normalization of secondary observers. The main concern in the above papers of Lockwood et al. and Usoskin et al., however, has been that the methods in question can lead to inflation of the resulting sunspot number (e.g., Usoskin et al. 2016b) while the problems we identify for the Hoyt and Schatten time series lead to an underestimate of group counts.

Our analysis is presented in Section 2 and the results are summarized and discussed in Section 3.

\section{Analysis}

\section{1. $k$-Factor time series}

Hoyt \& Schatten (1998a) considered 463 observers in their construction of GSN, with 373 beginning their observations in the year 1700 or later. The parameters for these observers are given in Appendix 1 of their paper, a portion of which is reproduced in Figure 2. Here we focus on the parameter in column 5 , the so-called $k^{\prime}$-factor ${ }^{2}$ that is used to scale secondary observers to the primary or reference observer used by Hoyt and Schatten - the Royal Greenwich Observatory (RGO) (Willis et al. 2013a, 2013b; Erwin et al. 2013; Willis et al. 2016a, 2016b). In the Hoyt \& Schatten (1998a, 1998b) observer normalization scheme, $k^{\prime}$-factors are formed by dividing the total number of sunspot groups observed by RGO by the corresponding total of the secondary observer, limiting the comparison to those days when both observers reported a non-zero group count. The comparison is made over the full period of overlap of the two observers, be it a fraction of

\footnotetext{
${ }^{2}$ In previous papers (Cliver \& Ling 2016; Cliver 2016), we have referred to these as $k$-factors, but following Wolf's and Hoyt and Schatten's lead, $k$-factor should be reserved for Wolf type sunspot numbers, with $k^{\prime}$ used for group numbers.
} 
E.W. Cliver: Sunspot number recalibration

\begin{tabular}{|c|c|c|c|c|c|c|c|c|}
\hline (1) & (2) & (3) & (4) & (5) & (6) & $\begin{array}{l}\text { (7) } \\
\text { No. }\end{array}$ & (8) & \multirow{33}{*}{$\begin{array}{l}\text { Daisy- } \\
\text { chaining }\end{array}$} \\
\hline Obs. & Start & End & Obs. & & & of Std./ & Obs. Name/ & \\
\hline No. & Year & Year & Days & $\mathrm{k}^{\prime}$ & $10 \leqq$ & Sec.Obs. & Location & \\
\hline$\overline{329}$ & $\overline{1871}$ & $\overline{1877}$ & $\overline{1530}$ & $0 . \overline{969}$ & $0 . \overline{0} 08$ & 2 & Secchi, Rome & \\
\hline 330 & 1872 & 1875 & 308 & 1.048 & 0.018 & 2 & Billwiller, Zürich & \\
\hline 331 & 1872 & 1874 & 282 & 1.211 & 0.068 & 2 & Sawyer, Cambridgeport & \\
\hline 332 & 1874 & 1976 & 37472 & 1.000 & 0.001 & 2 & Royal Greenwich Observatory & \\
\hline 333 & 1874 & 1893 & 3598 & 1.227 & 0.146 & 2 & Moncalieri & \\
\hline 334 & 1874 & 1875 & 107 & 1.170 & 0.064 & 2 & Main, Radcliffe Observatory & \\
\hline 335 & 1876 & 1879 & 997 & 0.838 & 0.008 & 2 & Billwiller \& Wolfer, Zürich & \\
\hline 336 & 1876 & 1882 & 1940 & 0.796 & 0.049 & 2 & Aguilar, Madrid & \\
\hline 337 & 1877 & 1886 & 2383 & 1.021 & 0.063 & 2 & Monthly Weather Review & \\
\hline 338 & 1880 & 1928 & 12536 & 1.094 & 0.016 & 2 & Wolfer & \\
\hline 339 & 1880 & 1892 & 3709 & 0.896 & 0.026 & 2 & Ricco, Palmero & \\
\hline 340 & 1882 & 1882 & 88 & 1.007 & 0.052 & 2 & Miethe, Potsdam & \\
\hline 341 & 1882 & 1910 & 6161 & 1.148 & 0.162 & 2 & Winkler, Jena & \\
\hline 342 & 1882 & 1887 & 1164 & 1.014 & 0.031 & 2 & Janesch, Laibach & \\
\hline 343 & 1883 & $18 \underline{96}$ & $32 \underline{21}$ & 0.997 & $\underline{0.000}$ & 2 & Merino, Madrid & \\
\hline$\overline{34} \overline{4}$ & $1 \overline{8} \overline{4}$ & $\overline{18} \overline{86}$ & $-9 \overline{65}$ & $1 . \overline{42} 9$ & $\overline{0.000}$ & 1 & Kokides, Athens & \\
\hline 345 & 1885 & 1905 & 3531 & 1.604 & 0.000 & 1 & Konkoly, Ogyalla & \\
\hline 346 & 1886 & 1886 & 162 & 1.392 & 0.000 & 1 & Vogel, Potsdam & \\
\hline 347 & 1886 & 1935 & 4534 & 1.329 & 0.000 & 1 & Stonyhurst College Obs. & \\
\hline 348 & 1887 & 1887 & 52 & 2.000 & 0.000 & 1 & Wilsing, Potsdam & \\
\hline 349 & 1888 & 1892 & 1359 & 1.180 & 0.000 & 1 & Schmoll, Paris & \\
\hline 350 & 1888 & 1899 & 2063 & 1.274 & 0.000 & 1 & Haverford College Obs., PA & \\
\hline 351 & 1888 & 1890 & 326 & 1.178 & 0.000 & 1 & Yendell, Boston & \\
\hline 352 & 1889 & 1921 & 10860 & 1.440 & 0.000 & 1 & Quimby, Philadelphia & \\
\hline 353 & 1889 & 1892 & 523 & 1.270 & 0.000 & 1 & Carleton College Observatory & \\
\hline 354 & 1889 & 1890 & 262 & 1.055 & 0.000 & 1 & Frost, Dartmouth & \\
\hline 355 & 1890 & 1891 & 258 & 1.056 & 0.000 & 1 & Smith Observatory & \\
\hline 356 & 1890 & 1890 & 67 & 1.040 & 0.000 & 1 & Hadden, Alta, Iowa & \\
\hline 357 & 1890 & 1890 & 9 & 1.273 & 0.000 & 1 & Furniss, Vassar & \\
\hline 358 & 1890 & 1925 & 2799 & 1.603 & 0.000 & 1 & Mount Holyoke College & \\
\hline
\end{tabular}

Fig. 2. Excerpt from Appendix 1 of Hoyt \& Schatten (1998a) which contains a list of the observers they considered along with their properties. Column descriptions: (1) Observer number; (2) and (3) First and last years of recorded observations; (4) the number of observing days; (5) $k^{\prime}$-factor; (6) the $1 \sigma$ uncertainty of the $k^{\prime}$-factor (based on multiple intercomparison paths before 1884); (7) the number of standard or secondary observers used to calculate the $k^{\prime}$-factor; (8) observer name and/or primary location where observations were made. The dashed blue line indicates the demarcation between observers who were scaled directly to RGO and those who were scaled to RGO by daisy-chaining.

one year or several decades. Group counts for a given day will vary from observer to observer because of such factors as telescope aperture and quality, visual acuity, seeing conditions, atmospheric transparency, and the observer's definition of a sunspot group. The $k^{\prime}$-factors adjust for these variables, bringing all observers to the same scale as RGO.

Figure 3 a contains a simple plot of the series of $k^{\prime}$-factors for Hoyt and Schatten observers with identification numbers (column 1 in Fig. 2) from 91 (J. Cassini) through 463 (British Astronomical Association). The endpoint observers in this sequence began observing in 1700 and 1992, respectively. ${ }^{3}$ On the $x$-axis of the plot the observers' identification numbers are indicated along with the first year during which every 20 th observer began recording group counts. Thus the $x$-axis represents an uneven time line because of the various circumstances that might cause anyone to begin observing the Sun to record sunspot groups. In the plot, red data points indicate observers in the Hoyt and Schatten database (http://www.ngdc.noaa.gov/stp/spaceweather/solar-data/solar-indices/sunspot-numbers/group/) who made observations for at least 100 days. Dashed vertical lines in the figure indicate the observing onsets of Schmidt (Observer No. 292) in 1841, Merino (No. 343) in 1883, and Batavia (No. 426) in 1920. Schmidt's observing onset is indicated because he was used as a secondary reference observer in the modified GSN, here designated GSN*, of Cliver \& Ling (2016). Merino is notable because he is the last observer, as one goes forward in time, who is not scaled directly to RGO, as indicated by a " 2 " in column 7 of Figure 2. After Batavia in

\footnotetext{
${ }^{3}$ We did not extend the plot back to 1610 because $k^{\prime}$-factors are poorly defined from 1610 to 1699 . Of the 90 observers during this period, 57 (including 17 of 34 with $\geq 100$ observations) have nominal Hoyt and Schatten $k^{\prime}$-factors of 1.255 , indicating that they had no non-zero days of overlap with any other observer.
}

$1920, k^{\prime}$-factors of new observers (i.e., observers who begin reporting groups in a given year or range of years) dropped abruptly. Average values of $k^{\prime}$-factors for observers who began counting groups in each of the four segments into which the $x$-axis is divided by the vertical dashed lines are as follows: (1) Nos. 91-291, 1.182; (2) Nos. 292-343, 1.075; (3) Nos. $344-426,1.431$; (4) Nos. 427-463, 1.166. ${ }^{4}$ These average values are indicated by horizontal dashed lines in Figure $3 \mathrm{a}$.

The plot in Figure 3a has several peculiar features: (a) The low 1.075 average $k^{\prime}$-factor of new observers from 1841 to 1883 in interval 2 is due to a high number of low- $k^{\prime}$-factor observers. Of the 51 new observers during this interval, 25 (49\%; not including RGO) had $k^{\prime}$-factors $\leq 1.02$, indicating that they were counting groups with a proficiency comparable to that of the modern RGO photographic patrol. (b) During interval 3, $k^{\prime}$-factors increase from a value of $\sim 1.2$ in 1884 to a value of $\sim 1.6$ in $\sim 1920$ (solid line linear fit in Fig. 3a), implying that new observers regressed relative to those of the previous epoch, and were, on average, counting groups at a progressively lower rate than RGO. (c) After 1920, in interval 4 , new observers have systematically lower $k^{\prime}$-factors than those at the end of the preceding interval (average $k^{\prime}$-factor of 1.166 vs. $\sim 1.6$ ).

We attribute the last of these peculiarities, the abrupt drop in $k^{\prime}$-factors after 1920 , to an apparent decision by Hoyt \& Schatten (1998a, 1998b) to populate their database primarily with low $(\sim 1.0-1.3) k^{\prime}$-factor observers after that year. Hoyt and Schatten write that after 1848 observations were plentiful

\footnotetext{
${ }^{4}$ Eight observers with outlying $k^{\prime}$-factors were omitted from the plot and the averages: five with values $>3.000$ (Nos. 175, 7.869; $217,5.111 ; 232,4.849 ; 265,3.051 ; 424,3.670)$, and three with values $<0.500$ (Nos. 146, 0.415; 150,0.399; 271, 0.451). None of these observers reported group counts for more than 30 days.
} 
(a)

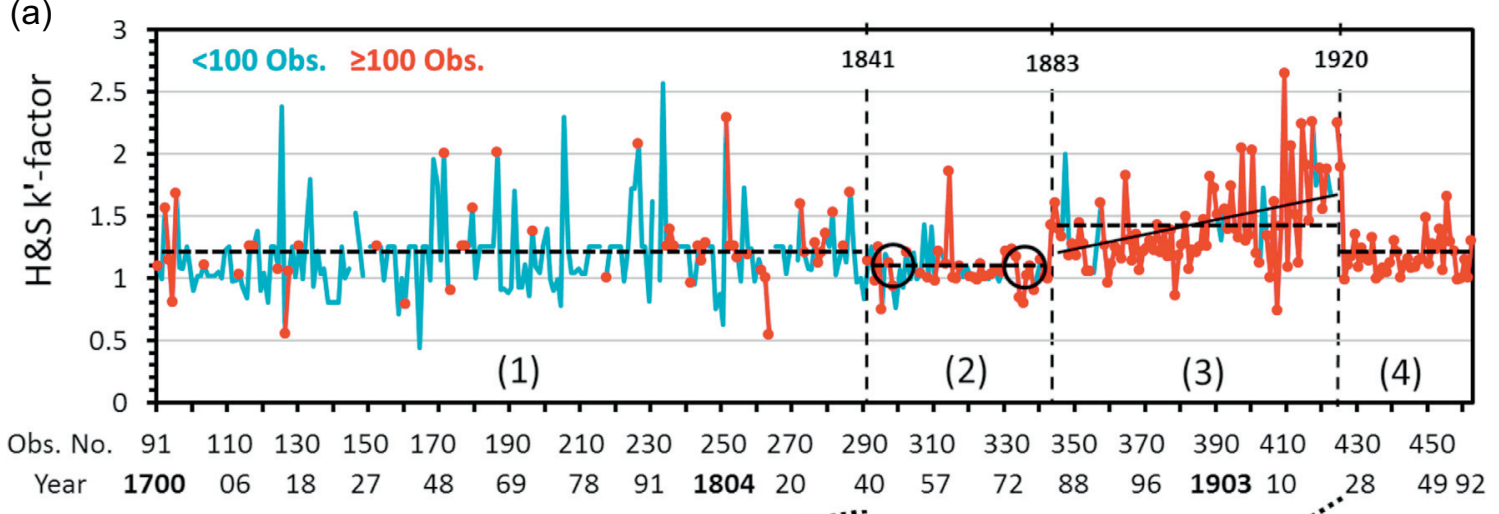

(b)

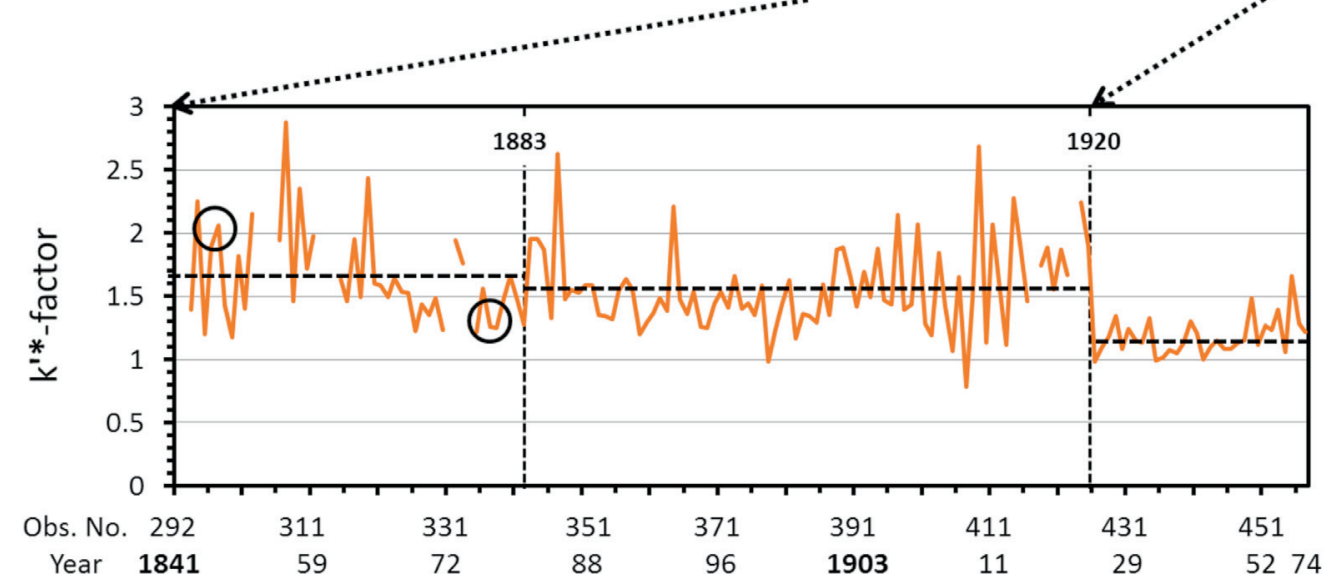

Fig. 3. (a) A plot of Hoyt \& Schatten (1998a) $k^{\prime}$-factors vs. the assigned number of the associated observer and the year that this observer began recording sunspot groups. Blue and red data points indicate observers who recorded sunspot groups for $<100$ and $\geq 100$ days, respectively. The time intervals and first and last observers for each of the four segments separated by the dashed vertical lines are as follows: (1) 1700-1840 (J. Cassini to Lohse); (2) 1841-1883 (Schmidt to Merino); (3) 1884-1920 (Kokides to Batavia); and (4) 1921-1992 (Mt. Wilson (Full Disk) to British Astronomical Association). Dashed horizontal lines give average values of the $k^{\prime}$-factors for observers who began counting groups during each interval (i.e., "new observers" during that interval). The slanted solid line in interval 3 indicates the increase in $k^{\prime}$-factors for new observers during this period. Black circles indicate the $k^{\prime}$-factors of Wolf (No. 298) and Wolfer (No. 338). The mean uncertainty in $k^{\prime}$ for Observers 91-343 is $\sim 0.1 * k^{\prime}$; thereafter it is zero (Hoyt \& Schatten 1998a; Appendix 1). (b) Same as (a) except here only intervals 2-4 are shown and revised $k^{\prime}$-factors ( $k^{*}$-factors) are taken from Cliver \& Ling (2016). The endpoint observers for interval 4 are Mt. Wilson (Full Disk) (1923) and Dogan (1974).

and they could afford to discard observers with $k^{\prime}$-factors $<0.6$ and $>1.4$.

\subsubsection{Long-term behavior of the $k^{\prime}$ series}

The two segments at the ends of the $k^{\prime}$-factor series in Figure 3a, segment 1 from 1700 to 1840 and segment 2 from 1921 to 1992 , have similar average values of 1.182 and 1.166 , respectively. This implies that the typical sunspot observer during the 18th century counted spots on par with his 20th century counterpart. Such constancy is not expected. In general, we would expect that the bulk of observers would gradually improve over time, i.e., count more groups relative to the standard RGO observer (for which, by definition, $k^{\prime}=1$ throughout the entire 1700-1992 interval), resulting in a decrease in average $k^{\prime}$ factors from segment 1 to segment 4 .

First, there is the expected gradual improvement in the telescopes used by sunspot observers over time. Consider Staudacher (or Staudach; Svalgaard \& Schatten 2016) - a key long-term observer during the second half of the 18th century (with 1142 observations from 1749 to 1799; Clette et al. 2014). Arlt (2008) writes that, "Nearly all of the spots [in Staudacher's drawings] show no distinction of umbra and penumbra. The size of the ink spots indicates that Staudacher included the penumbra in the spot size. He had certainly difficulties in separating them with his amateur telescope of the time. If he could not, he most likely missed all the A and B spot groups (referring to the Waldmeier classification; Waldmeier 1938)." In the Waldmeier classification, A and B spot groups account for $\sim 40 \%$ of all groups (Clette et al. 2014). Thus to convert Staudacher's counts to the modern scale, one must multiply them by a $k^{\prime}$-factor of $\sim 1.65$.

Comparison of Appendix 1 in Hoyt \& Schatten (1998a) with Table 1 in Svalgaard \& Schatten (2016) shows that Staudacher was one of a cohort of prolific (i.e., with at least 100 daily observations of sunspots during their observing lifetimes) "low group count" (requiring a large upward correction of daily group counts) observers during this period that included Zucconi (899 observations), Horrebow (1532 observations), Lalande (105), and Flaugergues (Viviers; 694). The only other observers with $\geq 100$ observations from 1749 to 1799 were Schubert ("high group count" observer; 492), Lievog (unspecified, 196), and Flaugergues (unspecified; C. De. T.; 130). Further inspection of the Hoyt and Schatten 
database confirms that "low group count" observers are dominant from 1749 to 1799 , with 4372 daily group observations by "low count" observers, 492 by "high count" observers, and $\sim 1000$ by unspecified observers during this period.

A second factor that would increase group counts over time for a given level of solar activity is an improvement in groupsplitting technique, which in the early days was based primarily on heliographic separation. Svalgaard (2016) has shown that modern group-splitting will increase Staudacher's counts (made by Wolf in the 19th century from Staudacher's drawings and subsequently used by Hoyt and Schatten) by an additional $25 \%$ (or 30\%; Usoskin et al. 2016a), yielding a net scaling $\left(k^{\prime}\right)$ (or correction) factor of $\sim 2$ relative to a modern reference observer.

Finally, there was a tendency, exemplified by Wolfer, who began observing in 1876, for new observers in the late 19th century and early 20 th century to count groups differently than had been done in the past. In contrast to Wolf (Wolfer's predecessor as the person responsible for producing the Zürich sunspot number), Wolfer included single "fine points and gray pores" in his counts of spot groups (Wolf 1857; Wolfer 1895; Kopecký et al. 1980). Wolf had difficulty seeing such groups because he used smaller telescopes. (In addition, he may have refrained from counting such groups to keep fidelity with earlier sunspot observers whose results he was collecting.) Thus, Wolfer counted 65\% more groups than Wolf for common days of observation during their 1876-1893 period of overlap (Cliver \& Ling 2016). The tendency for new observers toward the end of the 19th century to count more groups than their predecessors is supported by the fact that only 4 of 14 observers with at least 1000 observations in their careers (Hoyt \& Schatten 1998a; Appendix 1) that began counting groups during the $\sim 35$ years (1841-1876) before Wolfer began in 1876 had $k^{\prime}$-factors relative to Wolfer (Table 1 in Cliver \& Ling 2016) of $<1.2$ vs. 18 of 32 observers for the $\sim 45$ years (18771920) thereafter.

Each of the above effects (improvements in telescopes, tendency for new observers in the late 19th century to count more (smaller) groups than their predecessors, and refined groupsplitting techniques) should cause $k^{\prime}$-factors to increase as one goes back in time from the present. Figure 56 in Clette et al. (2014) indicates that the median $k$-factor for the $\sim 130$ observers in the modern Sunspot Index and Long-term Solar Observations (SILSO) network is $\sim 1.1$ (range from $\sim 0.8$ to $\sim 1.5$ ), well below the nominal value of $\sim 2$ for 18 th century observers such as Staudacher. The similar $k^{\prime}$-factors for new observers in the 18th and mid-20th century in Figure 3a point to a flaw (or flaws) in the Hoyt \& Schatten (1998a, 1998b) observer normalization scheme.

\subsubsection{The anomaly in the $k^{\prime}$ series from 1841 to 1920}

The quasi-progressive increase in $k^{\prime}$-factors for new observers from 1884 to 1920 (interval 3) corresponds to an inhomogeneity in the RGO reference series for the GSN that has been pointed out elsewhere (e.g., Clette et al. 2014; Cliver \& Ling 2016). Rather than the increase in $k^{\prime}$-factors during this interval being due to new secondary observers counting fewer groups relative to RGO, it is more plausibly caused by RGO counting progressively more groups relative to the actual level of solar activity. From the beginning of the RGO sunspot patrol in 1874 , we posit that RGO observers followed an effective institutional "learning curve" in counting groups due to presumed improvements in instrumentation and technique before stabilizing circa 1915. In a recent paper, Chatzistergos et al. (2017) note that that the time span over which the early RGO group count record is inhomogeneous is under debate and cite Sarychev \& Roshchina (2009) and Lockwood et al. (2016b) who presented evidence for much shorter intervals of nonuniformity of 1875-1880 and 1875-1877 (and 18921895), respectively. Neither Sarychev \& Roshchina (2009) nor Lockwood et al. (2016b), however, considered data for individual observers, other than Greenwich, beyond 1900.

The increase in early RGO group counts in comparison with those of other long-term observers can be seen in Figure 24 of Clette et al. (2014) for the average of 22 longterm observers from 1876 to 1928 and in Figure 2 of Cliver \& Ling (2016) in a direct comparison with Wolfer. To substantiate this result, we show in Figure 4 the rising ratio of yearly RGO group counts to annual average group counts based on the unscaled (i.e., $k^{\prime}=1$ ) daily group counts of all observers (omitting RGO) for each year from 1874 to 1920 (All UNSC $_{\text {( }}$ RGO), top trace with dashed-line linear fit). In contrast, the ratio of the yearly group count for Wolfer (a primary observer during this interval for both the Clette \& Lefèvre $\left(S_{N}{ }^{*}, 2016\right)$ and Svalgaard \& Schatten $\left(R_{\mathrm{GS}} ; 2016\right.$ time series) to the annual averages of unscaled daily group counts for all other observers (omitting Wolfer; $\operatorname{All}_{\mathrm{UNSC}}(-$ Wolfer), middle trace with linear fit) from 1876 to 1920 is relatively stable. The simplest explanation for this behavior, following the logic that Clette et al. $(2014,2016)$ used to identify the post-1980 Locarno drift, is that the RGO group count for a given level of solar activity is increasing during this period while, on average, all other observers are stable. The anomalous rise in $k^{\prime}$ factors from $\sim 1884$ to 1920 in Figure $3 \mathrm{a}$ is a telltale clue of the RGO inhomogeneity revealed by the corresponding rise in the top trace in Figure 4.

The complex, and flawed, daisy-chaining method used by Hoyt \& Schatten $(1998 \mathrm{a}, 1998 \mathrm{~b})^{5}$ to link secondary observers to RGO for years before 1884 (despite the fact that direct comparison with RGO was possible back to 1874) also contributes to the reduced $k^{\prime}$-factors before that year. We know that their procedure was flawed because of another telltale clue in Appendix 1 of Hoyt \& Schatten (1998a). Comparison of the $k^{\prime}$-factor of Wolf (1.117; Observer No. 298) given there is only $\sim 2 \%$ higher than that for Wolfer (1.094; No. 338) - with these values depicted by black circles in Figure $3 \mathrm{a}$ - despite the fact that Wolfer counted $65 \%$ more groups on common observing days. ${ }^{6}$

\footnotetext{
${ }^{5}$ Hoyt \& Schatten (1998a, p. 195) describe this procedure as follows: If the value of $k^{\prime}$ for a secondary or higher order observer is less than 0.6 or greater than 1.4 , that observer is not used for any intercomparisons. The value of $k^{\prime}$ for a tertiary observer is found by weighing their ratios to the secondary observer by the quality of the secondary observer [defined to be the number of intercomparison days divided by the quantity $\left(\left|\left(1-k^{\prime}\right)\right|\right)$ ]. The above process is repeated for the 4th, 5th, 6th, and 7th level observers [none of the 2nd through 7 th level (or beyond) observers are identified]. This technique maximizes the contribution of the best and most active observers and minimizes the number of intermediate observers between RGO and the observer for whom $k^{\prime}$ is being calculated. It utilizes all the information we have rather than a selected subset. Finally, because multiple intercomparison paths are followed, both the mean $k^{\prime}$ and its standard deviation can be calculated.

${ }^{6}$ This 1.65 ratio is non-linear and varies between a factor of $\sim 2$ for low ( $\sim 1$ group) activity and $\sim 1.2$ for active ( $\sim 10$ group) periods (see Fig. 7a in Usoskin et al. 2016a).
} 


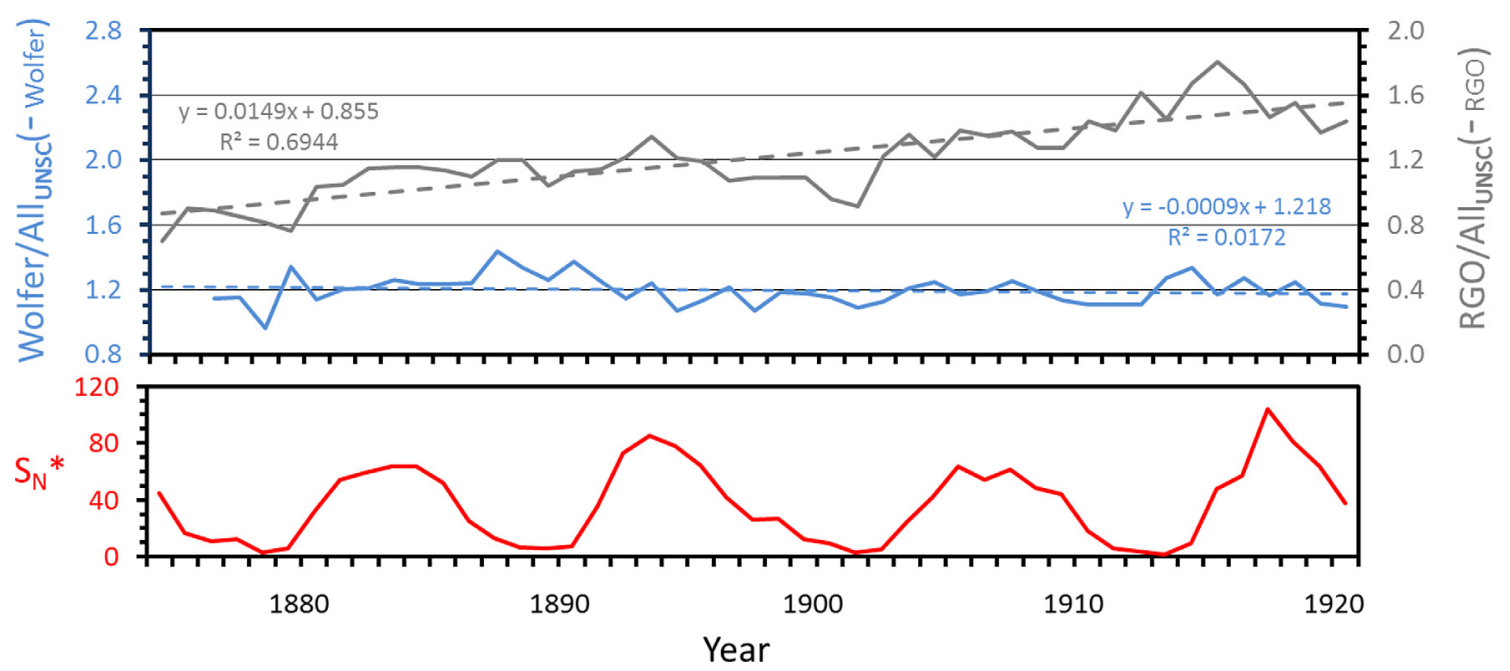

Fig. 4. Top curve. The ratio of annual RGO group counts to the yearly average of group counts of all observers (with RGO omitted; $\operatorname{All}_{\mathrm{UNSC}}(-\mathrm{RGO})$ ) from 1874 to 1920 . Middle curve. The ratio of annual Wolfer group counts to the yearly average of group counts of all observers (with Wolfer omitted; $\operatorname{All}_{\mathrm{UNSC}}(-$ Wolfer)) from 1876 to 1920 . Note the offset of the left and right $y$-axis scales. Bottom curve. Annual sunspot number $\left(S_{N}^{*}\right), 1874-1920$. Dashed-line linear fits are given for the two top traces. The Vaquero et al. (2016) database (http://www.sidc.be/silso/groupnumberv3) was used for this analysis (with Mt. Wilson (Center of Disk) eliminated).

The discontinuity between the abnormally low $k^{\prime}$-factors of interval 2 (average value of 1.075 for new observers between 1841 and 1883) and the rising $k^{\prime}$-factors of interval 3 occurs at precisely the point where Hoyt \& Schatten (1998a, 1998b) began to use daisy-chaining to link observers to RGO (indicated by the change from " 1 " to " 2 " in column 7 of Fig. 2). In addition to being the source of the downward jump in the $k^{\prime}$ (and GSN) series, as one goes back in time from 1884 to 1883, the daisy-chain link to earlier observers locks in the larger reduction in GSN due to the inhomogeneity in the reference RGO series.

Cliver \& Ling (2016) attempted to correct the flaws in the GSN by: (1) adjusting the 1874-1915 RGO daily group counts upward to give them a constant ratio on an annual basis relative to those of Wolfer over this interval; and (2) comparing the observations of secondary observers directly to adjusted RGO over the 1874-1915 interval and to Schmidt (Observer No. 292) scaled to the adjusted RGO for the 1841-1873 interval. $^{7}$ This produced the series of $k^{\prime *}$-factors shown in Figure $3 \mathrm{~b}$. In this figure, note the general lifting of $k^{*}$-factors for observers beginning before $\sim 1900$ which largely removes the puzzling low $k^{\prime}$-factors of interval 2 and rising $k^{\prime}$-factors of interval 3 in Figure $3 \mathrm{a}$. The black circles in Figure $3 \mathrm{~b}$ for Wolf (Observer No. 298) and Wolfer (No. 338) indicate $k^{\prime *}$-factors of 2.082 and 1.259 , respectively. The resulting Wolf/Wolfer ratio of these $k^{*}$-factors of 1.65 is close to the 1.58 value directly calculated from the Hoyt and Schatten database for days of overlap, on which both observers reported at least one group (Cliver \& Ling 2016). The revised $k^{*}$-factor time series in Figure $3 \mathrm{~b}$ is more in accord with the expected gradual improvement of secondary observers relative to a high-quality homogeneous standard observer over time, with an inferred characteristic $k^{*} *$-factor of $\sim 2$ during the 18th century (Sect. 2.1.1) followed by average $k^{*}$-factors of $1.651,1.565$, and 1.179 , in intervals 2,3 , and 4, respectively. The 1920 transition between intervals 3 and 4 is still abrupt because, in our opinion noted above, it reflects Hoyt and Schatten's choice to

\footnotetext{
${ }^{7}$ This mimics the backbone procedure of Svalgaard \& Schatten (2016). Only a single daisy-chain is required.
}

limit high $k^{\prime}$ observers after 1920. As shown in Figure 5, the resulting revised GSN time series, designated GSN*, more closely resembles the $S_{N}{ }^{*}$ series of Clette \& Lefèvre (2016) and the $R_{\mathrm{GS}}$ backbone-based group sunspot number series of Svalgaard \& Schatten (2016) than the original GSN. Based on comparison with $R_{\mathrm{GS}}$, Cliver \& Ling (2016) concluded that their GSN* series overcorrected by $\sim 8 \%$ for cycles 12 and 13 , while the $S_{N}$ series was $\sim 10 \%$ too low, relative to $R_{\mathrm{GS}}$, at the maxima of cycles 10,12 , and 13 . Cycle 10 is affected by the Schwabe-Wolfer transition (Leussu et al. 2013; Clette et al. 2014; Clette \& Lefèvre 2016), and Cliver \& Ling (2016) (see Fig. 4 in Clette et al. 2014) called attention to the averaging of the observations of Wolf and Wolfer as a potential cause of depressed values of WSN during cycles 12 and 13 (and therefore for $S_{N}$ as well since no correction was applied to WSN for this interval; Clette \& Lefèvre 2016).

\subsection{Correction-factor time series}

Cliver (2016) introduced the concept of a "correction-factor" series that can be used generally to check the validity of any new sunspot number time series. The correction-factor series for a given sunspot number time series is defined by the ratio of annual averages of the group counts for that sunspot number to annual averages of the raw (unscaled) group counts for all observers. These raw averages are computed by first averaging the daily values of group counts for all observers for a given day, and then obtaining monthly and yearly averages, in turn. For the unscaled data series (see Fig. 1 in Svalgaard \& Schatten 2016), all observers are treated equally, i.e., $k^{\prime}$-factors are defined to be 1.000 for all observers. Thus for any year $(i)$ in the correction-factor (CF) time series for a given sunspot number time series, the $\mathrm{CF}$ value is given by

$$
\mathrm{CF}_{i}\left(S_{N}\right)=\mathrm{SNG}_{i} / \mathrm{UNSC}_{i}
$$

where $\mathrm{SNG}_{i}=$ the annual group count corresponding to the sunspot number for year $i$ and $\mathrm{UNSC}_{i}=$ the average of unscaled group counts for all observers for year $i$.

The CF series shows how the raw group count data was changed to produce the sunspot number time series under 


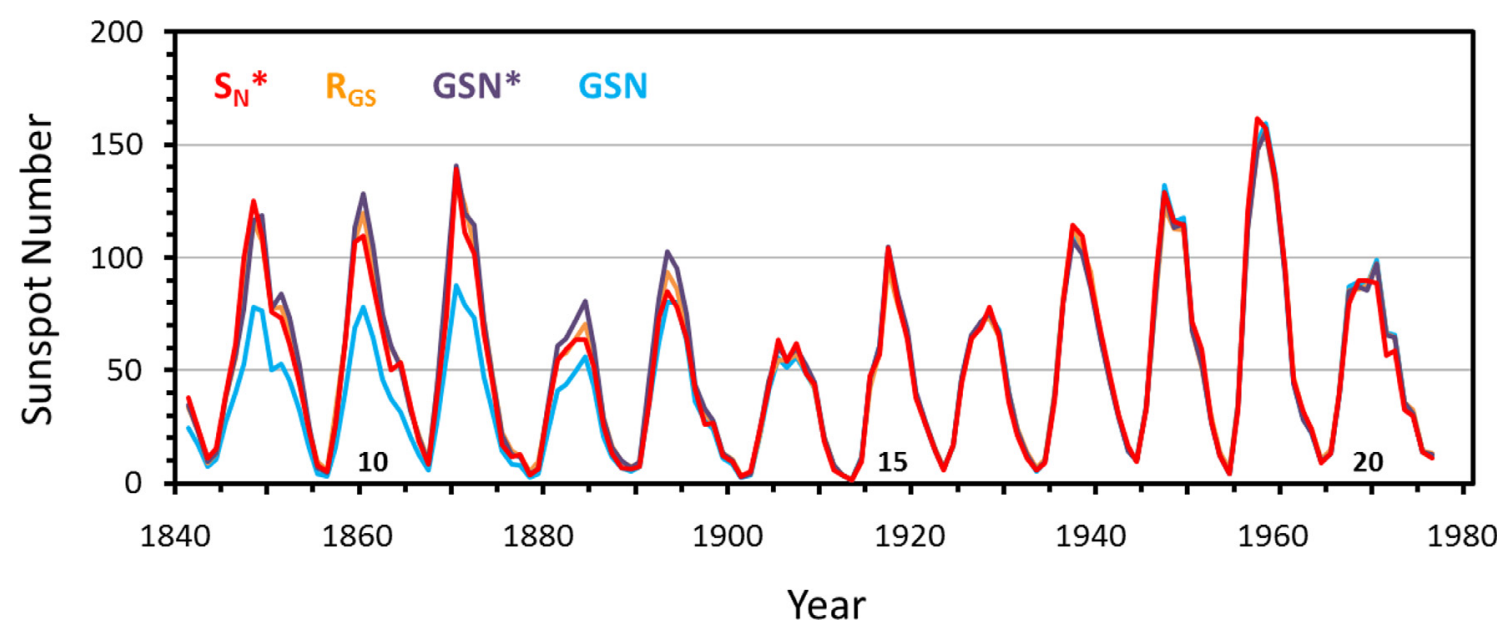

Fig. 5. Comparison of the original Hoyt \& Schatten (1998a, 1998b) GSN with the $S_{N}^{*}$ series of Clette \& Lefèvre (2016), the backbone-based group sunspot number $\left(R_{\mathrm{GS}}\right)$ of Svalgaard \& Schatten (2016), and the provisional GSN* series of Cliver \& Ling (2016) which corrected flaws in GSN, for 1841-1976. The three group sunspot number series are normalized to $S_{N}{ }^{*}$ over the 1916-1946 interval. Cycle numbers are given at the bottom of the figure.

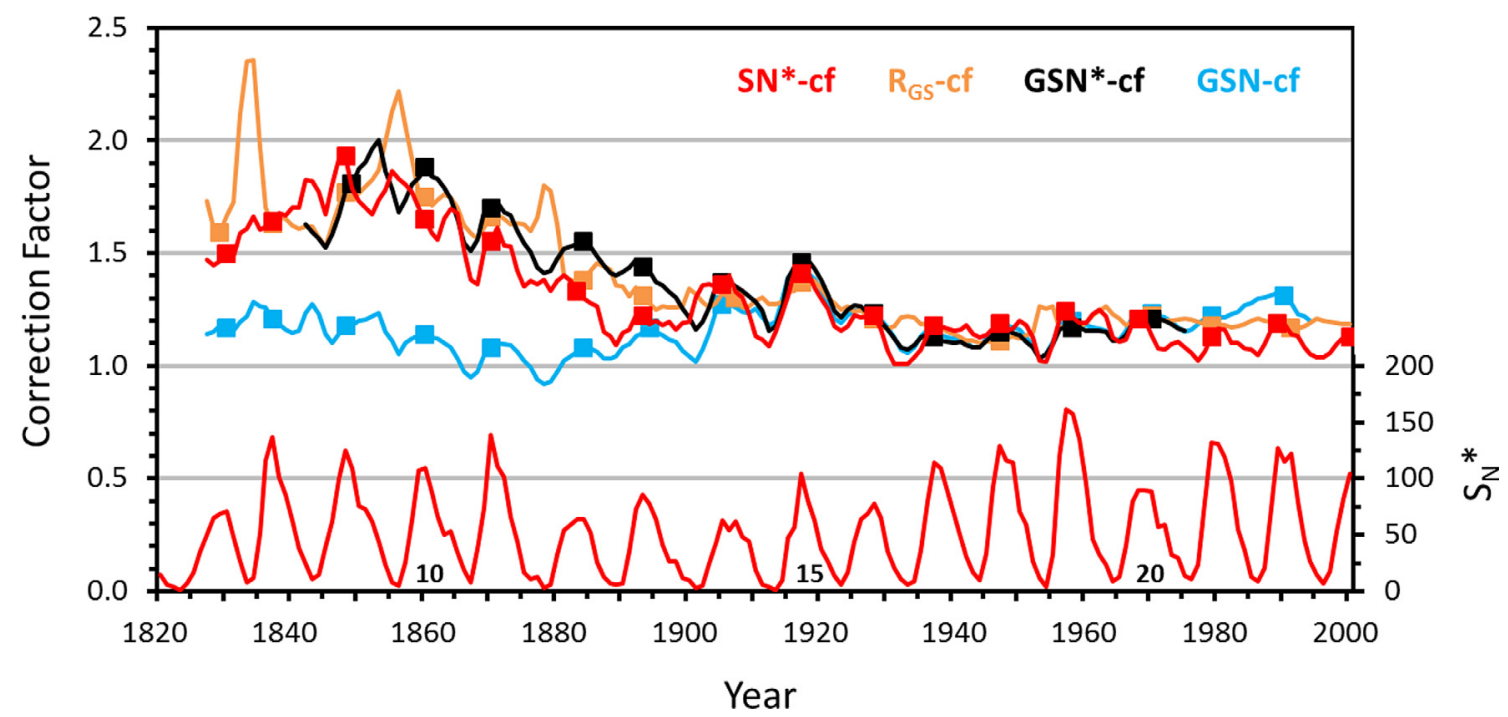

Fig. 6. Three-year running averages for 1826-2000 of correction-factor (CF) time series (Cliver 2016) for the four sunspot number time series in Figure 5. Years of maxima are indicated by square symbols and cycle numbers are given at the bottom of the figure.

consideration. Provided that observers are ample, the CF series can be used to identify both short-term and long-term anomalies in a sunspot number time series. A minimum of $\sim 5$ observers per day on average should suffice; this condition was met by the Hoyt and Schatten database for each of the decades during the 1860-1989 time period. A large number of observers gives the $\mathrm{CF}$ series a certain rigidity because the random addition or subtraction of individual observers over time is unlikely to cause a significant change in the denominator on the right-hand side of equation (1). On the other hand, an abrupt change in the numerator due to an inhomogeneity in the primary reference series will be readily apparent. Also, since the CF serves as a type of composite $k^{\prime}$-factor, we should expect the series to gradually decrease over time and converge to a low value near the presumed $k^{\prime}=1.000$ of the standard modern observer.

In Figure 6, we plot the 3-yr smoothed CF series for the four sunspot number time series in Figure 5 for the interval from 1826, when Schwabe began observing, to 2000. Square symbols indicate years of solar maxima for each of the series. The $S_{N}{ }^{*}$-cf, $R_{\mathrm{GS}}$-cf, and $\mathrm{GSN}^{*}$-cf series show the expected general decrease from the 19th to the 20th centuries as seen for the $k^{*}$-factors in Figure 3b. Figure 14 in Cliver (2016) shows the congruence between declining CF and average $k^{*}$-factors at the maxima of cycles 9-13 during the second half of the 19th century. In contrast, the relatively flat GSN-cf series in Figure 6 indicates little difference in the capability of the average observer to identify sunspot groups over the last two centuries, with observers on average counting slightly more groups relative to the modern standard during the 19th century $(<\mathrm{GSN}-\mathrm{cf}>=1.11)$ than in the 20th century $(<\mathrm{GSN}-$ cf $>=1.20$ ). The increase in $k^{\prime}$-factors from $\sim 1885$ to 1920 in Figure $3 \mathrm{a}$ is mimicked by the GSN-cf series in Figure 6, without the abrupt drop circa 1920. Rather the GSN-cf series decreases more gradually from $\sim 1.4$ at the peak of cycle 15 to an average value of $\sim 1.15$ for $\sim 1930-1965$ from which it rises 
(a)
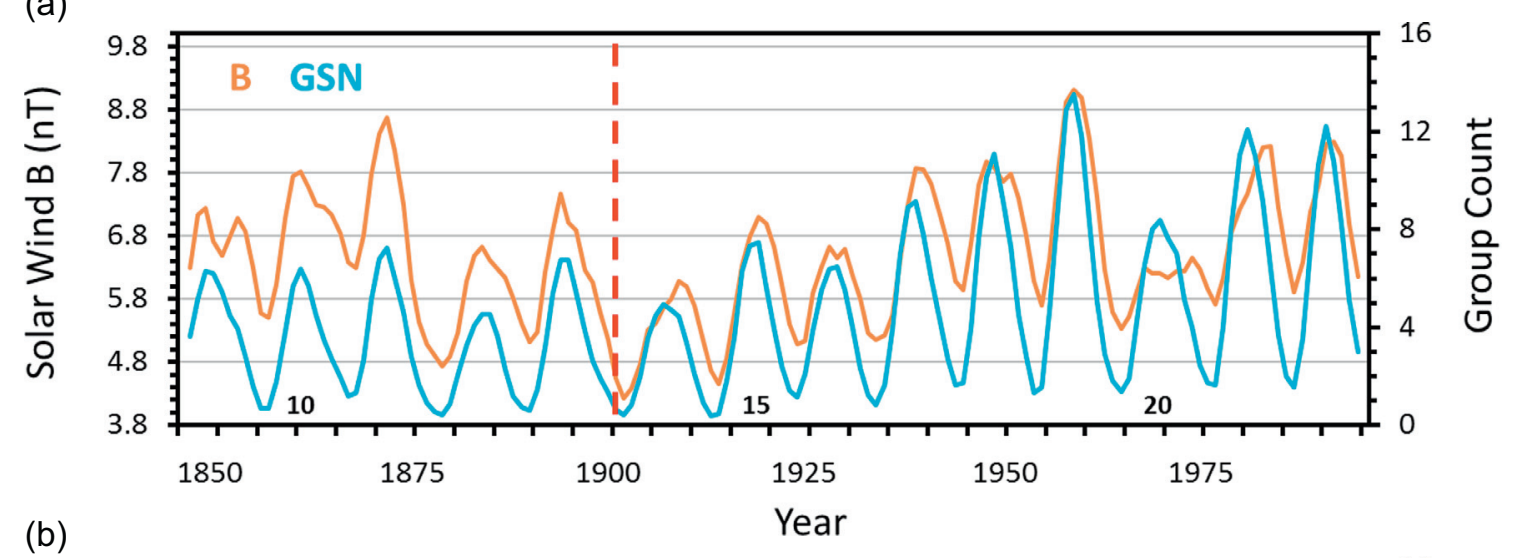

(b)

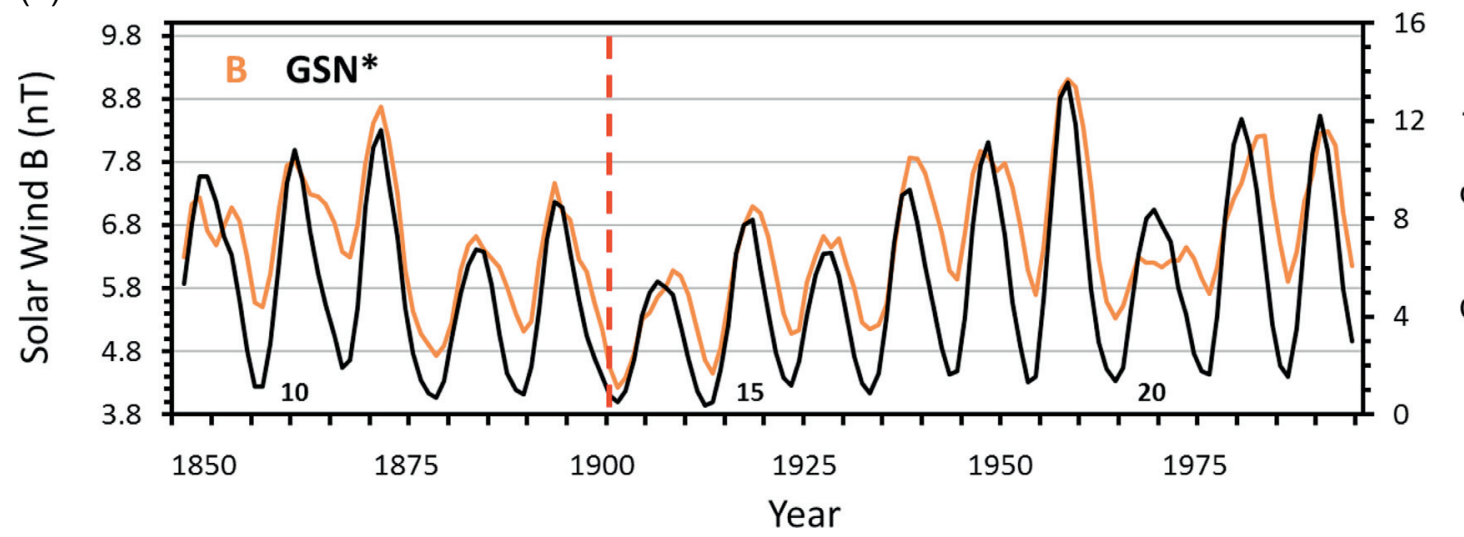

Fig. 7. Comparison of 3-yr smoothed GSN (a) and GSN* (b) sunspot number series with 3-yr smoothed solar wind $B$ from Owens et al. (2016; with observed data after 1964), 1845-1995. Solar cycle numbers are indicated at the bottom of the plots. In (a) and (b), the $B$ axis is offset by $3.8 \mathrm{nT}$ to match this parameter with 11-yr maxima of the sunspot number during the 20th century.

to $\sim 1.3$ at the maximum of cycle 22 . This late increase is attributed, at least in part, to the RGO-SOON transition after 1975 (Clette et al. 2014).

\subsection{Comparison of GSN* and GSN with solar wind $B$ (1845-2013)}

An additional check, not involving sunspot number data, can be applied to the GSN time series. Figures $7 \mathrm{a}$ and $7 \mathrm{~b}$ show comparisons of a recent construction of solar wind magnetic field strength ( $B$; Owens et al. 2016) with GSN and GSN*, respectively. Figure $7 \mathrm{a}$ shows that while $B$ agrees reasonably well with GSN at 11-yr peaks during the 20th century, this correspondence breaks down at cycle maxima during the $1800 \mathrm{~s}$, indicating that GSN is too low for that century. In comparison, the agreement between peak levels of GSN* and $B$ is reasonably consistent over the full 1845-1995 interval.

\section{Conclusion}

\subsection{Summary}

The observer normalization factors (termed $k^{\prime}$-factors) used to construct the group sunspot number (GSN) time series (Hoyt \& Schatten, 1998a, 1998b) contain an anomaly for observers who began observing from 1841 to 1920 : from 1841 to 1883 approximately half $(25 / 51)$ of all new observers had $k^{\prime}$-factors $\leq \sim 1.0$ while after 1883 the average $k^{\prime}$-factor progressively increased to a value $\sim 1.6$ circa 1920 (Fig. 3a). At face value, this implies an unusual number of exceptionally strong new observers from 1841 to 1883 that counted groups as adeptly as RGO, the standard observer, is assumed to have done over its full 1874-1976 observing period. Also, again at face value, the increase in $k^{\prime}$-factors from 1884 to 1920 implies a progressive decrease - for no apparent reason - in the capability of new observers to identify spot groups on the Sun. Rather, as shown elsewhere (e.g., Clette et al. 2014; Cliver \& Ling 2016), and substantiated in Figure 4, the Royal Greenwich Observatory (RGO) counted progressively more sunspot groups relative to other long-term-observers from 1874 to $\sim 1915$. Removing the inhomogeneity in the RGO time series, and using a more transparent normalization scheme than that employed by Hoyt \& Schatten (1998a, 1998b) for secondary observers prior to 1884 (Cliver \& Ling 2016), removes the $k^{\prime}$-factor anomaly (Fig. 3b) and brings the modified GSN time series, designated GSN*, into reasonable agreement with the new $S_{N}^{*}$ (Clette \& Lefèvre 2016) and $R_{\mathrm{GS}}$ (Svalgaard \& Schatten 2016) time series (Fig. 5). These findings are corroborated by a correction-factor (CF) time series (Cliver 2016) based on ratios of annual averages of group counts used to construct GSN (as well as GSN*, $S_{N}{ }^{*}$, and $R_{\mathrm{GS}}$ ) to annual averages of raw (un-normalized) group counts for all observers (Fig. 6), as well as by a comparison of the GSN and GSN* time series with a new reconstruction of solar wind $B$ (Owens et al. 2016) from 1845 to 2013 (Fig. 7). 


\subsection{Discussion}

The $\sim 1840-1920$ anomaly in the $k^{\prime}$-factors of the group sunspot numbers shown in Figure $3 \mathrm{a}$ is apparent on inspection of Appendix 1 of Hoyt \& Schatten (1998a) (an excerpt of which is shown in Fig. 2). Had the GSN series been more carefully scrutinized in 1998, it seems doubtful that the recent community effort to examine the sunspot number(s) would have occurred. In one sense, that would have been unfortunate. The detailed investigation of the Wolf and group sunspot number series has taught us much about both. For the WSN, these include the identification of the Schwabe-Wolf transition (cycle 10) (Leussu et al. 2013; Clette \& Lefèvre 2016), the Waldmeier discontinuity beginning after 1946 (Svalgaard 2012, Svalgaard et al. 2017), and the Locarno scaling drift (1981-2015) (Clette et al. 2014, 2016). In regard to the GSN, the Sunspot Number Workshops drew attention to the inhomogeneity in the early RGO series responsible for the $\sim 1885$ discontinuity (Svalgaard 2010; Clette et al. 2014; Cliver \& Ling 2016), the 1874-1976 interval used to scale GSN to WSN which was affected by both the RGO inhomogeneity and the Waldmeier discontinuity (Clette et al. 2014), and the RGO-ISOON transition after 1975 (Clette et al. 2014). Both series have benefitted from the important ongoing work of Vaquero, Arlt, and others (e.g., Vaquero 2007; Arlt 2008, 2009, 2011; Vaquero et al. 2011, 2012; Carrasco et al. 2013; Vaquero \& Gallego 2014, Diercke et al. 2015; Vaquero et al. 2016) to both critically evaluate and increase the sunspot Hoyt \& Schatten (1998a, 1998b) group number database, including digitization, archival, and accessibility. In addition, Clette et al. (2016) have specified a constant non-linear relationship between the sunspot number and group counts. Finally, the reexamination of the sunspot number and related long-term series has led to detailed investigation of the Maunder Minimum (e.g., Riley et al. 2015; Usoskin et al. 2015; Zolotova \& Ponyavin 2015) which will lead to a firmer view of solar activity during that intriguing low-activity period.

Thus in retrospect, the GSN, while flawed, has impelled a great deal of progress in our understanding of long-term solar variability. That said, it is unfortunate that the flaws in the GSN were not identified sooner. A good deal of work based, at least in part, on the GSN will now need to be reevaluated, the most notable of which is the concept of the Modern Grand Maximum, corresponding to the period of high peak sunspot numbers for cycles 18-22 ( 1945-1995) that is taken to be the strongest interval of activity in the past 1000 years or more (Solanki et al. 2004; Usoskin et al. 2007; Beer et al. 2012; Steinhilber et al. 2012; Usoskin 2013; cf., Muscheler et al. 2016). As Hoyt \& Schatten (1998a, 1998b) wrote in their abstract, "Solar activity before 1882 is generally lower than generally assumed [by $\sim 44 \%$ relative to WSN as noted above] and consequently solar activity in the last few decades is higher than it has been for several centuries." Recent papers such as that of Lockwood et al. (2016b), which characterize certain of the new reconstructions of solar activity as "radically different" from the GSN, miss the point that the Hoyt \& Schatten (1998a, 1998b) group sunspot number represented a similar departure from the original Wolf sunspot number. In contrast, the Clette \& Lefèvre (2016) and Svalgaard \& Schatten (2016) reconstructions are relatively conservative in the changes they propose, or imply in the case of Svalgaard and Schatten, for the original WSN.

The staid sunspot number has suddenly become a lively field of research. This is as it should be, given its widespread applications, e.g., solar dynamo studies, space weather, and terrestrial climate change. The lesson to be learned from reexamination of the original Hoyt \& Schatten GSN is that all new series need to be thoroughly investigated/examined/vetted. The good news is that, as the result of discussions at Space Climate 6 , the solar community is committed to doing just that in order to understand and, insofar as possible, reconcile the differences between the various sunspot number time series in Figure $1 \mathrm{~b}$. This reconciliation work has already begun.

Acknowledgements. I thank Frédéric Clette and Leif Svalgaard for helpful discussions and three referees (Mike Lockwood and two anonymous) for constructive comments. I thank Leif Svalgaard for assistance in constructing Figure 4. The editor thanks Mike Lockwood and two anonymous referees for their assistance in evaluating this paper.

\section{References}

Arlt, R. Digitization of sunspot drawings by Staudacher in 1749-1796. Sol. Phys., 247, 399-410, 2008, DOI: $10.1007 / \mathrm{s} 11207-007-9113-4$.

Arlt, R. The solar observations at Armagh Observatory in 1795-1797. Astron. Nachr., 330, 311-316, 2009, DOI: 10.1002 /asna.200911195.

Arlt, R. The sunspot observations by Samuel Heinrich Schwabe. Astron. Nachr., 332, 805-814, 2011, DOI: 10.1002 asna.201111601.

Beer, J., K.G. McCracken, and R. von Steiger. Cosmogenic radionuclides: theory and applications in the terrestrial and space environments, Springer, Berlin, ISBN: 978-3-642-14650-3, 2012.

Carrasco, V.M.S., J.M. Vaquero, M.C. Gallego, and R.M. Trigo. Forty two years counting spots: solar observations by D.E. Hadden during 1890-1931 revisited. New Astron., 25, 95-102, 2013, DOI: $10.1016 /$ j.newast.2013.05.002.

Chatzistergos, T., I.G. Usoskin, G.A. Kovaltsov, N.A. Krivova, and S.K. Solanki. New reconstruction of the sunspot group number since 1739 using the direct calibration and "backbone" methods. $A \& A, 2017$, in press.

Clette, F., and L. Lefèvre. The new sunspot number: assembling all corrections. Sol. Phys., 291, 2629-2651, 2016,

DOI: $10.1007 / \mathrm{s} 11207-016-1014-\mathrm{y}$.

Clette, F., L. Svalgaard, J.M. Vaquero, and E.W. Cliver. Revisiting the sunspot number. A 400-year perspective on the solar cycle. Space Sci. Rev., 186, 35-103, 2014 DOI: $10.1007 / \mathrm{s} 11214-014-0074-2$.

Clette, F., E.W. Cliver, L. Lefèvre, L. Svalgaard, and J.M. Vaquero. Revision of the sunspot number(s). Space Weather, 13, 529-530, 2015, DOI: 10.1002/2015SW001264.

Clette, F., L. Lefèvre, M. Cagnotti, S. Cortesi, and A. Bulling. The revised Brussels-Locarno sunspot number (1981-2015). Sol. Phys., 291, 2733-2761, 2016, DOI: 10.1007/s11207-016-0875-4.

Cliver, E.W. Comparison of new and old sunspot number time series. Sol. Phys., 291, 2891-2916, 2016, DOI: $10.1007 / \mathrm{s} 11207-016-0929-7$.

Cliver, E.W., and A.G. Ling. The discontinuity circa 1885 in the group sunspot number. Sol. Phys., 291, 2763-2784, 2016, DOI: $10.1007 / \mathrm{s} 11207-015-0841-6$.

Cliver, E.W., F. Clette, and L. Svalgaard. Recalibrating the Sunspot Number (SSN): the SSN workshops. Cent. Eur. Astrophys. Bull., 37 (2), 401-416, 2013.

Cliver, E.W., F. Clette, L. Svalgaard, and J.M. Vaquero. Recalibrating the Sunspot Number (SN): the 3rd and 4th SN workshops. Cent. Eur. Astrophys. Bull., 39, 1-19, 2015.

Diercke, A., R. Arlt, and C. Denker. Digitization of sunspot drawings by Spörer made in 1861-1894. Astron. Nachr., 336, 53-62, 2015, DOI: 10.1002/asna.201412138. 
Erwin, E.H., H.E. Coffey, W.F. Denig, D.M. Willis, R. Henwood, and M.N. Wild. The Greenwich photo-heliographic results (1874-1976): initial corrections to the printed publications. Sol. Phys., 288, 157-170, 2013, DOI: 10.1007/s11207- 013-0310-z.

Hoyt, D.V., and K.H. Schatten. Group sunspot numbers: a new solar activity reconstruction. Sol. Phys., 179, 189-219, 1998a, DOI: $10.1023 / \mathrm{A}: 1005007527816$.

Hoyt, D.V., and K.H. Schatten. Group sunspot numbers: a new solar activity reconstruction. Sol. Phys., 181, 491-512, 1998b, DOI: 10.1023/A:1005056326158.

Hoyt, D.V., K.H. Schatten, and E. Nesmes-Ribes. The one hundredth year of Rudolf Wolf's death: do we have the correct reconstruction of solar activity? Geophys. Res. Lett., 21, 2067-2070, 1994, DOI: 10.1029/94GL01698.

Kopecký, M., G.V. Kuklin, and B. Růžičková-Topolova. On the relative inhomogeneity of long-term series of sunspot indices. Bull. Astron. Inst. Czech., 31, 267-283, 1980.

Leussu, R., I.G. Usoskin, R. Arlt, and K. Mursula. Inconsistency of the Wolf sunspot number series around 1848. $A \& A, \mathbf{5 5 9}, \mathrm{A} 28$, 2013, DOI: 10.1051/0004-6361/201322373, 4 pp.

Lockwood, M., M.J. Owens, and L. Barnard. Centennial variations in sunspot number, open solar flux, and streamer belt width: 1. Correction of the sunspot number record since 1874 . J. Geophys. Res., 119, 5172-5182, 2014a, DOI: $10.1002 / 2014 J A 019970$.

Lockwood, M., M.J. Owens, and L. Barnard. Centennial variations in sunspot number, open solar flux, and streamer belt width: 2 . Comparison with the geomagnetic data. J. Geophys. Res., 119, 5183-5192, 2014b, DOI: 10.1002/2014JA019972.

Lockwood, M., M.J. Owens, L. Barnard, and I.G. Usoskin. Tests of sunspot number sequences: 3. Effects of regression procedures on the calibration of historic sunspot data. Sol. Phys., 291, 2829-2841, 2016a, DOI: 10.1007/s11207-015-0829-2.

Lockwood, M., M.J. Owens, L. Barnard, and I.G. Usoskin. An assessment of sunspot number data composites over 1845-2014. Astrophys. J., 824, 54, 2016b, DOI: $10.3847 / 0004-637 \mathrm{X} / 824 / 1 / 54,17 \mathrm{pp}$

Muscheler, R., F. Adolphi, K. Herbst, and A. Nilsson. The revised sunspot record in comparison to cosmogenic radionuclide-based solar activity reconstructions. Sol. Phys., 291, 3025-3043, 2016, DOI: 10.1007/s11207-016-0969-z.

Owens, M.J., E. Cliver, K.G. McCracken, J. Beer, L. Barnard, et al. Near-Earth heliospheric magnetic field intensity since 1750: 1. Sunspot and geomagnetic reconstructions. J. Geophys. Res., 121, 6048-6063, 2016, DOI: 10.1002/2016JA022529

Riley, P., R. Lionello, J.A. Linker, E. Cliver, and A. Balogh, et al. Inferring the structure of the solar corona and inner heliosphere during the maunder minimum using global thermodynamic magnetohydrodynamic simulations. Astrophys. J., 802, 105, 2015, DOI: $10.1088 / 0004-637 \mathrm{X} / 802 / 2 / 105,14 \mathrm{pp}$.

Sarychev, A.P. and E.M. Roshchina Comparison of three solar activity indices based on sunspot observations. Solar Sys. Res., 43, 151-157, 2009, DOI: 10.1134/S0038094609020087.

Steinhilber, F., J.A. Abreu, J. Beer, I. Brunner, and M. Christl, et al. 9,400 years of cosmic radiation and solar activity from ice cores and tree rings. Proc. Natl. Acad. Sci. USA, 109 (16), 5967-5971, 2012, DOI: 10.1073/pnas.1118965109.

Solanki, S.K., I.G. Usoskin, B. Kromer, M. Schüssler, and J. Beer Unusual activity of the Sun during recent decades compared to the previous 11,000 years. Nature, 431, 1084-1087, 2004, DOI: 10.1038 /nature02995.

Svalgaard, L. Updating the Historical Sunspot Record. In: S.R., Cranmer, J.T. Hoeksema, and J.L. Kohl, Editors. Understanding a peculiar solar minimum, ASP Conference Series 428. San Francisco: Astron. Society Pacific, pp. 297-305, 2010.
Svalgaard, L. How well do we know the sunspot number? In: C.H., Mandrini, and D.F. Webb, Editors. Comparative Magnetic Minima: Characterizing Quiet Times in the Sun and Stars, IAU Symposium, vol. 286, 27-33, Cambridge: Cambridge University Press, 2012, DOI: 10.1017/S1743921312004590.

Svalgaard, L.A. Recount of sunspot groups on Staudach's drawings. Sol. Phys., 292, 4, 2016, DOI: 10.1007/s11207-016-1023-x, 9 pp.

Svalgaard, L., and K.H. Schatten. Reconstruction of the sunspot group number: the backbone method. Sol. Phys., 291, 2653-2684, 2016, DOI: 10.1007/s11207-015-0815-8.

Svalgaard, L., M. Cagnotti, and S. Cortesi. The effect of sunspot weighting, Sol. Phys., 292, 34, 2017, DOI: $10.1007 / \mathrm{s} 11207-016-1024-9,19 \mathrm{pp}$.

Usoskin, I.G. A history of solar activity over millenia. Living Rev. Sol. Phys., 10, 1, 2013, DOI: 10.12942/1rsp-2013-1.

Usoskin, I.G., S.K. Solanki, and G.A. Kovaltsov. Grand minima and maxima of solar activity: new observational constraints. $A \& A$, 471, 301-309, 2007, DOI: 10.1051/0004-6361:20077704.

Usoskin, I.G., R. Arlt, E. Asvestar, E. Hawkins, and M. Käpylä, et al. The Maunder minimum (1645-1715) was indeed a grand minimum: a reassessment of multiple datasets. $A \& A, \mathbf{5 8 1}, \mathrm{A} 95$, 2015, DOI: 10.1051/0004-6361/201526652, 19 pp.

Usoskin, I.G., G.A. Kovaltsov, M. Lockwood, K. Mursula, M. Owens, and S.K. Solanki. A new calibrated sunspot group series since 1749: statistics of active day fractions. Sol. Phys., 291, 2685-2708, 2016a, DOI: 10.1007/s11207-015-0838-1.

Usoskin, I.G., G.A. Kovaltsov, and T. Chatzistergos. Dependence of the Sunspot-group size on the level of solar activity and its influence on the calibration of solar observers. Sol. Phys., 291, 3793-3805, 2016b, DOI: 10.1007/s11207-016-0993-z.

Vaquero, J.M. Historical sunspot observations: a review. Adv. Space Res., 40, 929-9411, 2007, DOI: 10.1016/j.asr.2007.01.087.

Vaquero, J.M., and M.C. Gallego. Reconstructing past solar activity using meridian solar observations: the case of the Royal Observatory of the Spanish Navy (1833-1840). Adv. Space Res., 53, 1162-1168, 2014, DOI: 10.1016/j.asr.2014.01.015.

Vaquero, J.M., M.C. Gallego, I.G. Usoskin, and G.A. Kovaltsov.Revisited Sunspot data: a new scenario for the onset of the maunder minimum. Astrophys. J., 731, L24, 2011, DOI: $10.1088 / 2041-8205 / 731 / 2 / \mathrm{L} 24,4$ pp.

Vaquero, J.M., R.M. Trigo, M.C. Gallego, and F. DominguezCastro. Improving sunspot records: solar drawings of the late 19th Century from the Royal Astronomical Observatory of Lisbon. Observatory, 132, 376-384, 2012.

Vaquero, J.M., L. Svalgaard, V.M.S. Carrasco, F. Clette, L. Lefèvre, M.C. Gallego, R. Arlt, A.J.P. Aparicio, J.-G. Richard, and R.A. Howe. Revised collection of sunspot group numbers. Sol. Phys., 291, 3061-3074, 2016, DOI: 10.1007/s11207-016-0982-2.

Waldmeier, M. Chromosphärische Eruptionen. I. Zeitschrift für Astrophysik, 16, 276-290, 1938, ADS: http://adsabs.harvard.edu/ abs/1938ZA.....16..276W

Willis, D.M., H.E. Coffey, R. Henwood, E.H. Erwin, D.V. Hoyt, M.N. Wild, and W.F. Denig. The Greenwich photo-heliographic results (1874-1976): summary of the observations, applications, datasets, definitions and errors. Sol. Phys., 288, 117-139, 2013a, DOI: $10.1007 / \mathrm{s} 11207-013-0311-\mathrm{y}$.

Willis, D.M., R. Henwood, M.N. Wild, H.E. Coffey, W.F. Denig, E.H. Erwin, and D.V. Hoyt. The Greenwich photo-heliographic results (1874-1976): procedures for checking and correcting the sunspot digital datasets. Sol. Phys., 288, 141-156, 2013b, DOI: $10.1007 / \mathrm{s} 11207-013-0312-x$.

Willis, D.M., M.N. Wild, and J.S. Warburton. Re-examination of the daily number of sunspot groups for the royal observatory, Greenwich (1874-1885). Sol. Phys., 291, 2519-2552, 2016a, DOI: $10.1007 / \mathrm{s} 11207-016-0856-7$. 
E.W. Cliver: Sunspot number recalibration

Willis, D.M., M.N. Wild, G.M. Appleby, and L.T. Macdonald. The Greenwich photo-heliographic results (1874-1885): observing telescopes, photographic processes and solar images. Sol. Phys., 291, 2553-2586, 2016b, DOI: 10.1007/s11207-016-0894-1.

Wolf, R. Sonnenflecken Beobachtungen in der zweiten Hälfte des Jahres 1850. Mitt. Nat. Forsch. Ges. Bern, 207, 89-95, 1851.

Wolf, R. Beobachtungen der Sonnenflecken in den Jahren 1849-1855. Astron. Mitteil. Eidgn. Sternw. Zürich, 1, 3-13, 1856.
Wolf, R. Beobachtungen über die Sonnenflecken im Jahre 1856. Astron. Mitt. Zürich, 3, 31, 1857.

Wolfer, A. Beobachtungen der Sonnenflecken im Jahre 1894. Astron. Mitt. Zürich, 86, 190, 1895.

Zolotova, N.V., and D.I. Ponyavin. The Maunder minimum is not as grand as it seemed to be. Astrophys. J., 800, 14, 2015, DOI: $10.1088 / 0004-637 \mathrm{X} / 800 / 1 / 42,14 \mathrm{pp}$.

Cite this article as: Cliver EW. Sunspot number recalibration: The $\sim 1840-1920$ anomaly in the observer normalization factors of the group sunspot number. J. Space Weather Space Clim., 7, A12, 2017, DOI: 10.1051/swsc/2017010. 